\title{
FORMACIÓN DOCENTE EN COMPETENCIAS CON ENFOQUE HUMANISTA PARA ADAPTARSE AL CAMBIO
}

\section{TEACHER EDUCATION IN COMPETENCES WITH A HUMANIST APPROACH TO ADAPT TO CHANGE}

Karina Trejo Sánchez

Doctora, Maestra y Licenciada en Derecho,
UNAM. Licenciada en Psicología, UNAM.
Profesora Investigadora Titular «C», Universidad
Autónoma Metropolitana, Unidad Cuajimalpa.
ktrejo@correo.cua.uam.mx

\section{RESUMEN}

El ensayo contiene una propuesta de competencias con enfoque humanista que podrían considerarse en programas de formación docente de todos los niveles educativos, toda vez que éstas se vislumbran necesarias para afrontar el constante cambio en los distintos ámbitos de la vida. El planteamiento de referencia tiene sustento teórico en las cuatro pilares del saber - vivir juntos, conocer, hacer y ser-, saber, saber hacer, saber ser y saber vivir juntos, esbozadas en el Informe a la UNESCO de la Comisión Internacional sobre la Educación para el Siglo XXI (Informe Delors, 1996). Toda vez que ante a los numerosos desafíos del porvenir, éste reconoce a la educación como un 
instrumento indispensable para que la humanidad pueda progresar hacia los ideales de paz, libertad y justicia social.

Palabras clave: cambio, competencias, educación, formación docente, humanismo, UNESCO.

\begin{abstract}
The essay describes a proposal for the development of competences with a humanist approach that considers programs for training teachers of all educational levels, since they are necessary to face today's constant change in all aspects of life. The argument departs within the framework set in Unesco's Delors report (1996), on Education for the Twenty First Century, based on the four pillars of learning, emphasizing the following types of knowledge: Learning to know, learning to do, learning to be and learning to live together. A blueprint for educational reform indispensable for humanity to progress towards the ideals of peace, freedom and social justice.
\end{abstract}

Key words: Change, competences, education, teachers' training, UNESCOS's, humanist approach.

\title{
I. INTRODUCCIÓN
}

La realidad conocida es sustituida rápidamente por otra y esto ocurre de manera frecuente en diversos ámbitos de la vida. Esta trasformación de la realidad constituye el cambio al que deben enfrentarse los educadores y para el cual se requiere considerar una formación docente enfocada en el desarrollo de las cualidades fundamentales del ser humano, y por ende, de la sociedad. Si los docentes se preparan en esta perspectiva, contarán con competencias - herramientas cognitivas, afectivas y sociales- para afrontar dicho cambio y estarán en posibilidad de trasmitirlas y fomentarlas en sus alumnos, y con ello, contribuir a que éstos desarrollen de mejor manera sus potencialidades, cultivando una mejor sociedad en la que se procure la existencia de la paz, la libertad y la justicia. 
El objetivo del presente escrito es presentar una propuesta de diversas competencias con perspectiva humanista que se considera indispensable abordar en los programas de formación docente de todos los niveles educativos, con la finalidad de preparar a los educadores para realizar su labor frente a los cambios vertiginosos que se dan en los distintos ámbitos de la vida. La OCDE (2016) ha reconocido, como las principales tendencias del cambio en los aspectos socioeconómico y político a: la globalización, el futuro del Estado-Nación, la interrogante respecto de que si las ciudades son los nuevos países, la reestructura familiar y las nuevas tecnologías. Los docentes requerimos ampliar nuestras competencias con aquellas que viabilicen nuestro actuar en estos contextos.

La competencias docentes que se propone incluir en la formación docente, parten del análisis de los cuatro pilares del conocimiento o vías del saber - vivir juntos, conocer, hacer y ser-, presentados en el Informe a la UNESCO de la Comisión Internacional sobre la Educación para el Siglo XXI (1996), el cual se eligió como fundamento teórico, debido a que su eje rector es la concepción de la función esencial de la educación en el desarrollo continuo de la persona y las sociedades, como una vía al servicio de un desarrollo humano más armonioso y genuino; lo cual es aplicable al proceso de formación docente, toda vez que éste tiende al perfeccionamiento personal y profesional del maestro.

El método seguido para el desarrollo del estudio es el análisis de los cuatro pilares del conocimiento, anteriormente mencionados, con el propósito de revelar su significado y características, a fin de plantear a través de ellas, una moción de competencias de corte humanista, necesarias en la formación docente de todos los niveles educativos para hacer frente al cambio.

\section{LA FORMACIÓN DOCENTE ANTE EL CAMBIO}

La sociedad está en constante cambio y la educación debe adecuarse para responder a las demandas que ésta le exige. Los docentes, como actores clave de la educación, estamos llamados a seguir formándonos 
continuamente, a actualizarnos y perfeccionarnos en pro de nuestro óptimo cometido, a fin de repercutir de manera positiva en el desarrollo de las potencialidades de nuestros alumnos y en la mejora continua de la sociedad.

\section{Principales tendencias que tienen el potencial de influir en el futuro de la educación}

La OCDE (2016) reconoce las capitales tendencias que tienen el potencial de influir en el futuro de la educación e indica los retos apremiantes a los que los actores que participan en la educación deberán responder, incluyendo a los formadores de docentes y en consecuencia, a éstos. Las tendencias de referencia que prevén cambios en la educación y que urgen a la adquisición de diversas competencias con enfoque humanista son:

A. La globalización, entendida como la ampliación, profundización y aceleración de las conexiones a través de las fronteras nacionales, como una creciente internacionalización de los mercados de bienes y servicios, medios de producción, sistemas financieros, competencia, empresas, tecnología e industrias. Esto requerirá un nuevo enfoque de las políticas económicas, pero también un nuevo énfasis amplio en la cohesión social. La educación posee un papel que desempeñar al proporcionar las habilidades y competencias necesarias para operar en este nuevo mundo (OECD, 2016: pp. 10 y 41).

B. El futuro del Estado-Nación, enfocado en el papel central que el Estado sigue teniendo para garantizar el bienestar y la seguridad de sus ciudadanos, desarrollando la productividad de su fuerza de trabajo y garantizando que el país disponga del talento necesario. En este sentido, los educadores deben ser conscientes de las habilidades que sus estudiantes necesitarán para prosperar en un mercado de trabajo más intensivo en conocimiento, así como el impacto potencial de cambios en la seguridad, la sanidad y las prioridades de gasto (OECD, 2016: p. 2).

C. La interrogante respecto de que si las ciudades son los nuevos países, en razón de su crecimiento poblacional. Algunos argumentan que las 
ciudades son ahora el nivel de gobernanza más relevante: lo suficientemente pequeñas como para reaccionar con respuestas rápidas a los problemas y, asimismo, lo suficientemente grandes como para controlar el poder económico y político. Las condiciones de vida de una ciudad se pueden mejorar y la educación puede y debe desempeñar un papel en todo esto, formando en valores cívicos y proporcionando los conocimientos suficientes para la participación comunitaria, apoyando la creatividad y la innovación de los ciudadanos (OECD, 2016: pp. 10 y 11).

D. La familia, ha sufrido diversas transformaciones. En los últimos cincuenta años, el número de familias mixtas y hogares monoparentales ha aumentado. Las familias son cada vez más pequeñas y las personas deciden tener hijos más tarde o no tenerlos. El número de divorcios va en aumento, aun cuando las tasas de matrimonio disminuyen. A medida que nuestro concepto de la institución del matrimonio se transforma, también lo hace nuestra concepción sobre las familias y las estructuras familiares. Entre los aspectos de la familia en que la educación puede contribuir, se encuentran abordar las diferencias en los valores sociales dentro de las escuelas y promover el respeto entre los estudiantes; promover la confianza entre padres, maestros y administradores; enseñar tolerancia y pensamiento crítico, y prevenir la radicalización (OECD, 2016: pp. 3 y 92).

E. Las nuevas tecnologías han cambiado la forma en que nos comunicamos, trabajamos e incluso socializamos y tienen el potencial de hacer más: las innovaciones en biotecnología, por ejemplo en la secuenciación del genoma, pueden revolucionar nuestras vidas. El ritmo del desarrollo tecnológico es exponencial y su impacto, a menudo, impredecible. La educación debe contribuir con una alfabetización mejorada que permita un mejor empleo de las nuevas tecnologías, lo cual conlleva a la necesidad de preparar al docente sobre este tema (OECD, 2016: pp. 98 y 110).

Dichos cambios exigen competencias de índole cognitivo, afectivo y social, «nos exige comprender mejor al otro, comprender mejor el mundo. Exigencias de entendimiento mutuo, de diálogo pacífico y, por qué no, de armonía» (Delors, J., 1996: p. 16). 


\section{Formación docente ante el cambio}

Los programas de formación buscan que los docentes optimicen su labor. Según el Diccionario de la Lengua Española (RAE, 2001) formar significa: «Dar forma a algo; criar, educar, adiestrar [...] adquirir desarrollo, aptitud o habilidad». De ahí que formarse «tiene que ver con la posibilidad de actuar y reflexionar para perfeccionar esa forma» (Barraza, 2001, p. 142). En estos términos, podemos entender por formación el «proceso permanente de adquisición, estructuración y reestructuración de conductas para el desempeño de una determinada función» (De Lella, C., 2003, p. 21), lo cual implica que la formación «hace referencia a un proceso amplio que debe insertarse en lo reflexivo, en el conocimiento de campos del saber que dan cuenta de lo educativo: la filosofía, psicología, pedagogía, etcétera» (Díaz Barriga, 1988, p. 176).

Por su parte, la formación docente «se ubica en el terreno de la educación formal donde el currículo establece sus linderos en la escuela, pensada como el medio que crea la sociedad para trasmitir la cultura, reproducir una forma de organización social y formar un sujeto para el desempeño de un puesto de trabajo» (Rivas, 2004, p. 58). Esto indica que los docentes podríamos incrementar nuestros talentos y potencialidades si participamos continuamente en procesos de formación, porque el maestro, como cualquier otro profesional, «aísla un problema, lo plantea, concibe y elabora una solución y asegura su aplicación en una institución y contexto determinados» (Pavié, 2011, p. 3); por tanto, debe estar altamente calificado, a fin de responder a los cambios que se le presentan y a las referidas exigencias sociales.

Para Peters, Guy B. (2003), las instituciones identifican las circunstancias cambiantes de su entorno y luego se adaptan a ellas (p. 57). Los docentes, como parte de los actores de las instituciones educativas, reconocemos la existencia de esos cambios, y como respuesta a ellos, debemos estar en constante formación, porque «[...] las demandas de la sociedad plantean desafíos a la cambiante naturaleza de la labor docente y a su desarrollo profesional» (Aguerrondo, I. y Pogré, P., 2001: 23). 
El desafío principal de la sociedad es preparar a las generaciones más jóvenes para las demandas futuras. Esto enfatiza la idea que las escuelas y los sistemas educativos no son fines en sí mismos, sino medios para que los estudiantes sean competentes para la vida, no sólo para la escuela (OCDE, 1999: p. 21).

\section{La importancia de la formación docente en la educación a lo largo de la vida}

Y en virtud de que el Informe a la UNESCO de la Comisión Internacional sobre la Educación para el Siglo XXI, señala que la educación durante toda la vida se presenta como una de las llaves de acceso al siglo XXI y responde al reto de un mundo que cambia rápidamente (Delors, J., 1996: p. 21), los docentes estamos obligados — por un deber ético y social- a hacer de la formación una tarea permanente.

Los integrantes de la Comisión de referencia, acentúan la necesidad de volver a la escuela para afrontar las novedades que surgen en la vida privada y en la vida profesional, y señalan que la única forma de satisfacerla es que todos aprendamos a aprender (Delors, J., 1996: p. 21). Además, sostienen que «la educación a lo largo de la vida debe dar a cada individuo la capacidad de dirigir su destino en un mundo en que la aceleración del cambio, acompañada del fenómeno de mundialización, tiende a modificar la relación de hombres y mujeres con el espacio y el tiempo» (Delors, J., 1996: p. 113). De ahí que la formación docente puede estar fundamentada en la importancia de la educación a lo largo de la vida, toda vez que ésta permitirá a los maestros afrontar los constantes cambios.

\section{LAS COMPETENCIAS Y SUS FORMAS DE MANIFESTACIÓN}

Conforme a Tobón (2005), existen diversas nociones sobre el vocablo competencia, atendiendo a diversos aspectos, entre ellos: la historia del término (del latín competere, encontrarse con, pertenecer a, rivalidad); 
la historia del concepto (filosófica, lingüística, sociológica, psicológica y de educación técnica); y a su estructura (proceso complejo, saber hacer, saber ser, problemas, actividades, responsabilidad, trasformación del contexto, manejo de la incertidumbre) (p. 48). En virtud de ello, aludiremos a algunas con el fin de tener un marco referencial al respecto.

Así, se entiende por competencia un conjunto denso, complejo, integrado y dinámico de saberes conceptuales, procedimentales y actitudinales que un ser humano ha conseguido desarrollar a ciertos niveles de calidad; y que le hacen apto para seguir aprendiendo (significativa, funcional y permanentemente). Esencialmente, hacen al sujeto competente para realizarse humanamente, socialmente y profesionalmente (Achaerandio Zauzo S.J., L., 2010: p. 11).

Como principio de la organización de la formación, la competencia puede apreciarse en el conjunto de actitudes, conocimientos y habilidades específicas que posibilitan llevar a cabo un trabajo o de resolver un problema en particular (Ouellet, 2000: 37).

Para (Bogoya, 2000), las competencias son una actuación idónea que emerge en una tarea concreta, en un texto con sentido, donde hay un conocimiento asimilado con propiedad y el cual actúa para ser aplicado en una situación determinada, de manera suficientemente flexible como para proporcionar soluciones variadas y pertinentes (p. 11).

En contraposición al concepto referido, para la OCDE (2010), una competencia es más que solo conocimiento: implica satisfacer demandas complejas, aprovechando y movilizando recursos psicosociales (p. 6). La competencia es un concepto más amplio que incluye capacidades, habilidades y actitudes. Así, una competencia es la forma en que las personas logran movilizar todos sus recursos personales (cognitivos, afectivos, sociales) para lograr el éxito en la resolución de una tarea en un contexto definido (Otero, J.M. y Luengo Orcajo, F., 2011, p. 33).

Para Gómez (1997), las competencias incluyen una intención (interés por realizar mejor las cosas, interés por hacer algo original), una acción 
(fijación de objetivos, responsabilidad sobre resultados, asunción de riesgos calculados) y un resultado (mejora en la calidad, productividad, ventas e innovación en servicios y productos) (p. 52).

Del interés por hacer algo original es que se advierte que «una competencia es una capacidad para el desempeño de tareas relativamente nuevas, en el sentido de que son distintas a las tareas de rutina que se hicieron en clase o que se plantean en contextos distintos de aquéllos en los que se enseñaron» (Vasco, 2003: 37).

Así, al ser las competencias «una compleja estructura de atributos necesarios para el desempeño de situaciones específicas» (Gonczi y Athanasou, 1996: 82), sus distintas formas de manifestación son: actitudes, capacidades y habilidades a desempeñar.

En razón de que las competencias implican «la capacidad adaptativa, cognitiva y conductual para responder adecuadamente a las demandas que se presentan en el entorno» (Frade Rubio, L., 2009: 82), la formación en competencias se requiere para enfrentar confiadamente el cambio, debido a que «hace del aprendizaje una práctica continua donde la gente aprende también a adaptarse al cambio» (Venegas Jiménez, P., 2011: 12).

Para Perrenoud (2004) una competencia es «la facultad de movilizar un conjunto de recursos cognitivos para solucionar con pertinencia y eficacia una serie de situaciones» (p. 11). Así, las formas de manifestación de las competencias docentes son: conocimientos, capacidades, habilidades y actitudes. Los cuales se definen a continuación:

Conforme a Rivas, T., Martín, C. y Venegas, N.A. (2003), el docente al tomar decisiones en la complejidad de su práctica, acude a distintos conocimientos que va construyendo a lo largo del proceso de formación docente, considerando las interpretaciones de las situaciones en las que se ve envuelto (p. 31).

Para los autores de referencia, el conocimiento del docente es de dos órdenes: científico y cotidiano, o de sentido común. El primero, «se caracteriza por ser explícito, formal, preciso, con una lógica coherente 
y consistente, explicativo y comprensivo de aspectos de la realidad, que permiten de alguna manera ordenarla y preverla. Es una construcción social de una determinada comunidad científica, inacabada y provisoria, en permanente reelaboración» (Rivas, T., Martín, C. y Venegas, N.A., 2003: p. 31). El segundo, «es la capacidad de las personas de adaptarse a su medio y a las normas que rigen dentro del mismo, mediante su comportamiento [...], es un proceso constante que se da en el transcurso de la vida y es independiente a cada persona» (Rivas, T., Martín, C. y Venegas, N.A., 2003: p. 31).

Según Compagnucci, E. y Cardós, P. (2007), también existe un conocimiento profesional que es una categoría referida al saber teórico y práctico del área de conocimiento del docente (p. 2).

Toda vez que el marco teórico de nuestra propuesta de competencias con enfoque humanista para adaptarse al cambio son los cuatro pilares del conocimiento o vías del saber - vivir juntos, conocer, hacer y ser-, presentados en el Informe a la UNESCO de la Comisión Internacional sobre la Educación para el Siglo XXI (1996), consideramos que la conceptualización más apegada a éstos es la propuesta por Gallego (1999) en torno a las competencias, las cuales señala: son procesos complejos que las personas ponen en acción-actuación-creación para resolver problemas y realizar actividades (de la vida cotidiana y del contexto laboral profesional), aportando a la construcción y trasformación de la realidad, para lo cual integran el saber ser (automotivación, iniciativa y trabajo colaborativo con otros), el saber conocer (desempeño basado en procedimientos y estrategias), teniendo en cuenta los requerimientos específicos del entorno, las necesidades personales y los procesos de incertidumbre, con autonomía intelectual, conciencia crítica, creatividad y espíritu de reto, asumiendo las consecuencias de los actos y buscando el bienestar humano. Las competencias en tal perspectiva están constituidas por procesos subyacentes (cognitivo-afectivos), así como también por procesos públicos y demostrables, en tanto implican elaborar — con rigurosidad — algo de sí, para los demás (p. 14).

Por su parte, las capacidades se desarrollan a partir de la experiencia histórico-social de la humanidad, mediante la transmisión de conocimientos y modos de actuar propagados a través de la actividad y la 
comunicación por diferentes generaciones; no son innatas. Se forman en estrecha relación con el proceso de adquisición de conocimientos (Méndez, A.G. y Salas, Morales J. F., 2003: 15 y 16).

Según Trianes Torres, M. V., y Gallardo Cruz, J. A. (2008), las habilidades son rutinas que existen para llevar a cabo tareas específicas; se desarrollan mediante técnicas que, a través del entrenamiento y la práctica, conducen a adquirir destreza o pericia en diversos dominios (p. 444).

En lo concerniente a las actitudes, De la Mora Ledesma (1990) señala que éstas: «son ideas con un tono emocional dirigido hacia o contra algo; suponen una tendencia a actuar de determinada manera» ( $\mathrm{p}$. 31). Y para Díaz-Barriga Arceo (2010) son: «experiencias subjetivas (cognitivo-afectivas) que implican juicios evaluativos, que se expresan en forma verbal o no verbal, que son relativamente estables y que se aprenden en el contexto social» (p. 45).

\section{FORMACIÓN DOCENTE EN COMPETENCIAS CON ENFOQUE HUMANISTA PARA ADAPTARSE AL CAMBIO}

No obstante que ya existen trabajos valiosos sobre propuestas de competencias docentes, este texto se centra en una moción con visión hondamente humanista y social, para la formación docente de todos los niveles educativos, dado que se encuentra fundamentada en los cuatro pilares del conocimiento, reconocidos por la Comisión Internacional sobre la Educación para el Siglo XXI en su Informe a la UNESCO (1996). Esto en virtud de que dicho Informe, proclama un enfoque humanista y social de la educación como proceso holístico, que liga la adquisición de conocimientos a la práctica y equilibra las competencias individuales y colectivas (Tawil, S. y Cougoureux, M., 2013, p. 7).

En el presente apartado se expondrán esas cuatro vías del saber y sus correlativas competencias en sus distintas formas de manifestación, que pueden ser: actitudes, capacidades y habilidades a desempeñar. 
Así, los cuatro aprendizaje fundamentales, «son necesarios para que los seres humanos puedan sobrevivir, desarrollar plenamente sus capacidades, vivir y trabajar con dignidad, participar plenamente en el desarrollo, mejorar la calidad de su vida, tomar decisiones fundamentadas y continuar aprendiendo» (Delors, J., 1996: p. 24). Y al extraer de estas vías del saber, las competencias básicas que a través de la formación docente puede desplegar el maestro, se contribuye tanto a su desarrollo personal y profesional como al de sus alumnos.

En el Informe de referencia (Delors, J., 1996), se enfatiza especialmente uno de los cuatro pilares del conocimiento: aprender a vivir juntos, en virtud de que la educación tiene la responsabilidad particular de contribuir al nacimiento de un nuevo humanismo, con un componente ético esencial (p. 53).

Sobre aprender a vivir juntos, se señala que implica conocer mejor a los demás, su historia, sus tradiciones y su espiritualidad. Y a partir de ahí, crear un espíritu nuevo que impulse la realización de proyectos comunes o la solución inteligente y pacífica de los inevitables conflictos, y se advierte que los otros tres pilares proporcionan los elementos básicos para aprender a vivir juntos (Delors, J., 1996, p. 22).

De ahí que aprender a conocer «tiende al dominio de los instrumentos mismos del saber a adquirir los instrumentos de la comprensión»; por su parte, aprender a hacer permite «obtener no sólo una calificación profesional sino, más generalmente, una competencia que capacite al individuo para hacer frente a gran número de situaciones»; y aprender a ser, implica que los seres humanos puedan «determinar por sí mismos qué deben hacer en las diferentes circunstancias de la vida» (Delors, J., 1996: pp. 96, 109 y 106).

Y para estar en posibilidad de presentar un planteamiento de las posibles competencias requeridas en la formación docente ante el cambio social, es necesario determinar las peculiaridades primordiales de los cuatro pilares del conocimiento. Así, entre las principales características para «aprender a vivir juntos» se encuentran: 
a. Tener actitud de empatía, lo cual sería fecundo para los comportamientos sociales a lo largo de la vida (Delors, J., 1996: p. 105); por tanto, una competencia al respecto radicaría en:

- Actitud de empatía. Consiste en adoptar el rol de la otra persona e imaginar la situación desde su perspectiva (Toribio Briñas, L., 2010: p. 33).

b. Desarrollar «la comprensión del otro» (Delors, J., 1996: p. 109), cuya competencia representaría:

- Actitud de comprensión al otro. Esta actitud valora y practica la comprensión mutua y la convivencia pacífica en el seno de una población mundial muy diversa (Luna Scott, C., 2015: p. 9).

c. Permite ayudar a transformar una interdependencia de hecho en la solidaridad deseada, implica realizar proyectos comunes, respetando los valores del pluralismo y la paz, y pasar de la tolerancia a una cooperación activa (Delors, J., 1996: pp. 5, 109 y 261). En este sentido, las competencias serían:

- Actitud valoral de solidaridad.

- Actitud valoral de respeto.

- Actitud valoral de pluralidad.

- Actitud valoral hacia la paz.

- Actitud valoral de tolerancia.

Las competencias éticas son valores esenciales que conducen a actuar correctamente. EI sentido ético es la capacidad para pensar, vivir $\mathrm{y}$ actuar conforme a los principios universales que se basan en el valor de la persona humana, y se dirigen a su pleno y maduro desarrollo (Achaerandio Zauzo S.J., L., 2010: pp. 6 y 27).

- Capacidad de cooperación. Supone desarrollar habilidades interpersonales, como la comunicación, el acuerdo en los objetivos de 
logro, cierta orientación hacia la tarea común, y un clima o ambiente social apropiado que cohesione al grupo o equipo, en torno a lo que es importante para lograr los objetivos comunes (Achaerandio Zauzo S.J., L., 2010: p. 28).

En lo tocante a las principales características de «aprender a conocer» están:

a. La capacidad de conocer implica construir conocimiento, previa la selección de la información pertinente (Achaerandio Zauzo S.J., L., 2010: p. 6). Y la competencia que de ahí se deriva es:

- Capacidad de construir conocimiento. Para Toribio Briñas, L. (2010), esta capacidad supone comprender opciones, formar opiniones, tomar decisiones y llevar a cabo acciones informadas y responsables. $Y$ requiere que los individuos:

- Reconozcan y determinen lo que no saben.

- Identifiquen, ubiquen y accedan a fuentes apropiadas de información.

- Evalúen la calidad, la propiedad y el valor de dicha información, así como sus fuentes.

- Organicen el conocimiento y la información.

b. «Implica tener en cuenta los rápidos cambios derivados de los avances de la ciencia y las nuevas formas de la actividad económica y social, porque la educación tiene que adaptarse en todo momento a los cambios de la sociedad» (Delors, J., 1996: pp. 22 y 23). A este respecto la competencia que de aquí subyace es:

- Capacidad de adaptación al cambio. Es la disposición para cambiar, crear y adaptarse en sintonía con un mundo cambiante y mejorable, supuesta la previa reflexión (Achaerandio Zauzo S.J., L., 2010: p. 31).

c. «En cuanto medio, consiste para cada persona en aprender a comprender el mundo que la rodea, al menos suficientemente para 
vivir con dignidad»(Delors, J., 1996: pp. 96 y 97). Por ende, la competencia que implica es:

- Capacidad de comprensión del entorno. Es la posibilidad para interactuar eficazmente con el mundo físico, tanto en sus aspectos naturales como en los generados por la acción humana, de tal modo que se posibilita la comprensión de sucesos, la predicción de consecuencias y la actividad dirigida a la mejora y preservación de las condiciones de vida propia, de las demás personas y del resto de los seres vivos. En definitiva, incorpora habilidades para desenvolverse adecuadamente, con autonomía e iniciativa personal, en ámbitos de la vida y del conocimiento muy diversos (salud, actividad productiva, consumo, ciencia, procesos tecnológicos, etcétera) y para interpretar el mundo (Toribio Briñas, L., 2010: p. 36).

Y como características de «aprender a hacer» tenemos:

a. Permite hacer frente a numerosas situaciones, algunas imprevisibles (Delors, J., 1996: p. 22). En este sentido la competencia que se devela es:

- Capacidad para afrontar y resolver conflictos. Parte de la base de que, para manejar efectivamente los inevitables conflictos con los que nos encontramos en todos los aspectos de la vida, hay que enfrentarlos, resolverlos y no negarlos, por lo que es necesario considerar los intereses y necesidades de otros y las soluciones en las que ambas partes ganen. Y acorde a Toribio Briñas, L. (2010), para ello, los individuos necesitarán poder:

- Analizar los elementos y los intereses en juego, los orígenes del conflicto y el razonamiento de todas las partes, reconociendo que hay diferentes posiciones posibles.

- Identificar áreas de acuerdo y áreas de desacuerdo.

- Recontextualizar el problema.

- Priorizar las necesidades y metas, decidiendo lo que están dispuestos a dejar de lado y bajo qué circunstancias (p. 33). 
Para Achaerandio Zauzo S.J., L. (2010), la resolución práctica de los problemas exige otras habilidades, destrezas y actitudes que hacen al ser humano no sólo «razonable y creativo», sino también eficiente y eficaz para ejecutar las acciones adecuadas, en el momento preciso; por eso se habla de investigación-acción como de un binomio interactivo de un pensamiento (que analiza la situación reflexivamente para tomar decisiones) y de una actividad operativa, con que el sujeto responde congruentemente a lo pensado y decidido (p. 29).

b. Combina la calificación propiamente dicha, adquirida mediante la formación y al trabajar en equipo (Delors, J., 1996: p. 100). De ahí que la competencia específica a este respecto es:

- Capacidad para trabajar en equipo. Conforme a Covey, Stephen R. (2000), el trabajo en equipo es una modalidad de articular las actividades de un grupo humano en torno a un conjunto de fines, metas y resultados a alcanzar. El trabajo en equipo implica una interdependencia activa entre los integrantes de un grupo que comparten y asumen una misión de trabajo. Implica la existencia de:

- Un objetivo, una finalidad o una meta común.

- Un grupo de personas comprometidas con esa convocatoria.

- Un grupo de personas con vocación de trabajar en forma asertiva y colaborativa.

- Una convocatoria explícita generadora de intereses movilizadores y de motivaciones aglutinantes.

- La construcción de un espacio definido por un saber-hacer colectivo (espacio donde se pueden identificar situaciones problemáticas, juzgar oportunidades, resolver conflictos, decidir acciones, llevarlas a cabo y evaluarlas). 
- Una comunicación fluida entre los miembros del equipo y su entorno.

- Una instancia efectiva para la toma de decisiones.

- Una red de conversaciones, comunicaciones e intercambios que contribuyen a concretar una tarea.

- Un espacio de trabajo dotado de las capacidades para dar cuenta de lo actuado (pp. 4 y 5).

c. Encierra adaptar la enseñanza al futuro mercado de trabajo (Delors, J., 1996: p. 99), con lo cual la competencia correspondiente radica en:

- Capacidad de innovación. Es la capacidad de «abrir nuevos caminos», aplicar maneras de pensar originales, proponer ideas y soluciones nuevas, plantear cuestiones desconocidas y llegar a respuestas inesperadas (Luna Scott, C., 2015: p. 12).

Y por último, entre las características de «aprender a ser» se advierten:

a. Que el ser humano se dote de un pensamiento autónomo y crítico, para elaborar un juicio propio y determinar por sí mismo qué debe hacer en las diferentes circunstancias de la vida (Delors, J., 1996: p. 106). En este entendido la competencia que esto entraña es:

- Capacidad de aprender a pensar. Como parte de «aprender a ser», se encuentra «aprender a pensar», la cual es una competencia fundamental, del punto de vista cognitivo, que entraña un pensamiento desarrollado (Achaerandio Zauzo S.J., L., 2010: p. 20). Es la capacidad de reflexión activa e investigadora. Implica el acceso a la información, su análisis y su síntesis, y puede enseñarse, practicarse y dominarse (Luna Scott, C., 2015: p. 5).

b. El fortalecimiento de la responsabilidad personal en la realización del destino colectivo (Delors, J., 1996: p. 23). A este respecto, la competencia es: 
- Capacidad de interdependencia. Implica los nexos con otros para la sobrevivencia material y psicológica, también en relación con la identidad social. Se hace importante manejar bien las relaciones interpersonales para beneficio de los individuos y para construir nuevas formas de cooperación (Toribio Briñas, L., 2010: p. 32).

c. La facilidad para comunicar con los demás (Delors, J., 1996: p. 23), en lo que la competencia resultante es:

- Habilidad de comunicación. La comunicación se define como la expresión con claridad de forma estructurada, con corrección y oportunidad, de las ideas, conocimientos y sentimientos propios, mediante la palabra hablada, adaptándose a las características del contexto y de los oyentes, para lograr su comprensión y aceptación (Achaerandio Zauzo S.J., L., 2010: p. 20). La OCDE (2010) señala que la comunicación juega un papel importante para preparar a los estudiantes no sólo como aprendices para toda la vida, sino también como miembros de una comunidad con sentido de la responsabilidad hacia los otros (p. 8).

d. Comprenderse mejor a uno mismo (Delors, J., 1996: p. 23) y la competencia al respecto es:

- Capacidad de autocomprensión. Es ser consciente de sus propias fortalezas, habilidades, actitudes y valores; esto tiene que ver con la competencia de «autoestima y automotivación: identidad»; se combina también con la llamada: «autoeficacia» (Achaerandio Zauzo S.J., L., 2010: p. 30). 
Cuadro 1. Competencias con enfoque humanista para adaptarse al cambio.
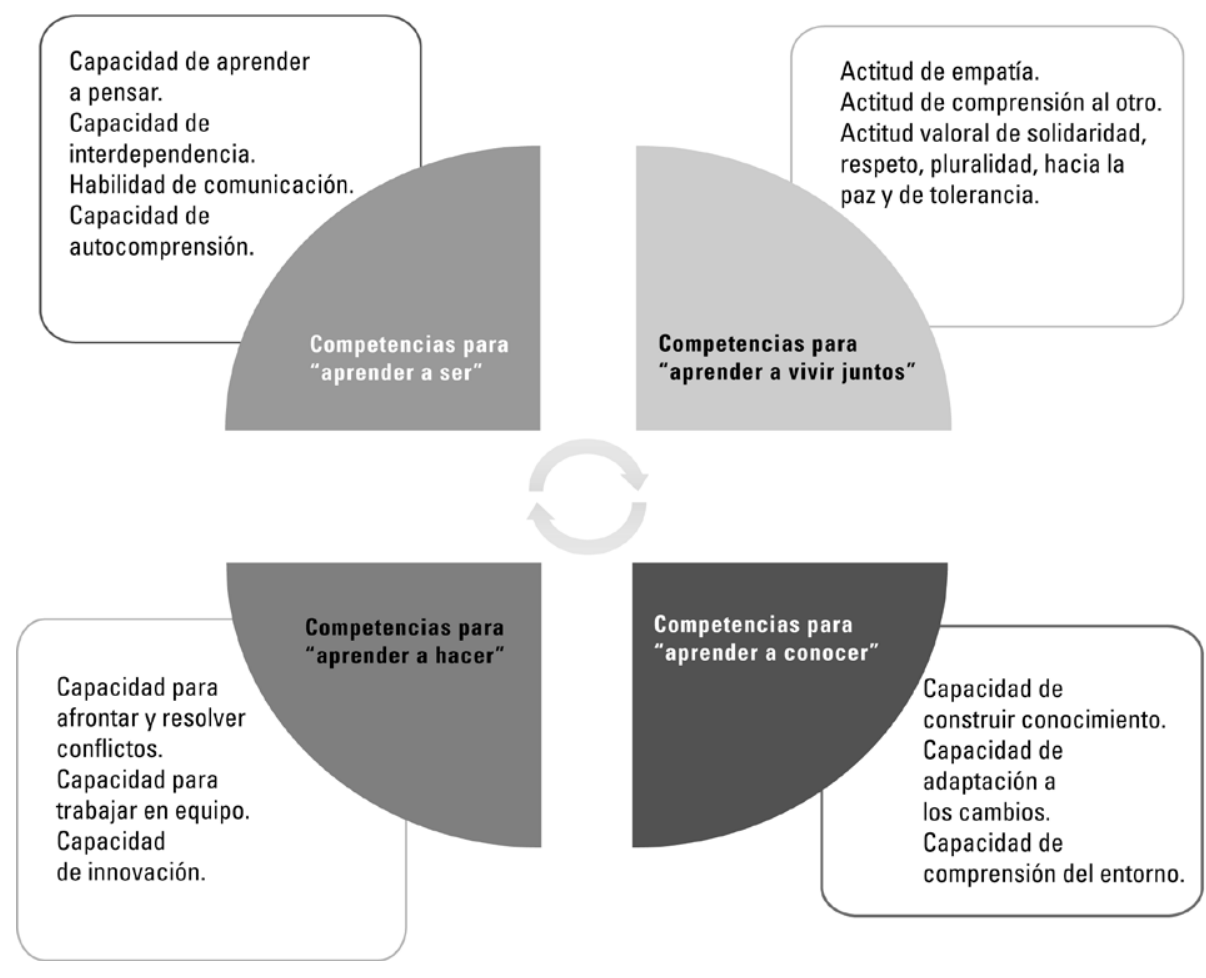

\section{CONCLUSIONES}

I. La formación es un proceso de aprendizaje permanente, en razón de que «la adquisición del conocimiento no concluye nunca y puede nutrirse de todo tipo de experiencias» (Delors, J., 1996: p. 99).

II. Los docentes deberíamos estar abiertos al aprendizaje y dotados de flexibilidad para prepararnos continuamente a fin de adquirir las nuevas competencias que se requieran, según los cambios del entorno. 
III. La formación docente en competencias con enfoque humanista dota de herramientas cognitivas, afectivas y sociales que permiten afrontar el cambio.

IV. Las competencias propuestas acopian elementos entre sí; no son excluyentes, sino se complementan, porque al estar sustentadas en las cuatro vías del saber, al igual que éstas, convergen en una sola, «ya que hay entre ellas múltiples puntos de contacto, coincidencia e intercambio», dado que como "el período de aprendizaje cubre toda la vida, cada tipo de conocimiento invade el ámbito de los demás y los enriquece» (Delors, J., 1996: pp. 95 y 112).

V. Las competencias presentadas son transversales, toda vez que cruzan diversos sectores de la cognición y de los ámbitos afectivo y social, porque en general, «las competencias tienden a favorecer el desarrollo de los niveles de pensamiento intelectual y a impulsar el crecimiento, y madurez de las actitudes y valores más elevados (Achaerandio Zauzo S.J., Luis, 2010: p. 15).

\section{REFERENCIAS}

Achaerandio Zauzo S.J., L. (2010). Competencias fundamentales para la vida, Guatemala: IGER.

Aguerrondo, I. y Pogré, P. (2001). Las instituciones de formación docente como centros de innovación pedagógica. Argentina: Troquel.

Barraza, A. (2007). La formación docente bajo una conceptualización comprehensiva y un enfoque por competencias. Revista Estudios Pedagógicos, n. 2. Chile: Universidad Austral de Chile.

Bogoya, D. (2000). Una prueba de evaluación de competencias académicas como proyecto, en Bogoya et al., Competencias y proyecto pedagógico, Bogotá: Universidad Nacional de Colombia. 
Compagnucci, E. y Cardós, P. (2007). El desarrollo del conocimiento profesional del profesor en psicología. Revista Orientación y Sociedad, n. 7. Argentina: Universidad Nacional de La Plata.

Covey, Stephen R. (2000). Trabajo en equipo, Buenos Aires: IIPE.

De la Mora Ledesma, J. G. (1990). Psicología del Aprendizaje. Formas 2. México: Progreso.

De Lella, C. (2003). Formación docente. Modelo hermenéuticoreflexivo y la práctica profesional. Revista Decisio. Otoño. Argentina: IDEAS.

De Rivas, T., Martín, C., Venegas, N.A. (2003). Conocimientos que intervienen en la práctica docente. Revista Praxis Educativa, n. 7. Argentina: ICEII.

Delors, J. (1996). Informe a la UNESCO de la Comisión Internacional sobre la Educación para el Siglo XXI. La educación encierra un tesoro. Madrid: UNESCO-Santillana.

Díaz Barriga, A. (1988). Investigación educativa y formación de profesores. En: Investigación educativa y formación de profesores. Contradicciones de una articulación. Cuadernos del CESU n. 20, México: UNAM.

Frade Rubio, L. (2009). Desarrollo de competencias en educación: Desde preescolar hasta bachillerato ( $2^{\mathrm{a}}$ ed.). México: Inteligencia educativa.

Gallego, R. (1999). Competencias cognoscitivas. Un enfoque epistemológico, pedagógico y didáctico. Bogotá: Cooperativa Editorial Magisterio.

Gómez, J. H. (1997). Mapa de competencias: Estrategias en el recurso humano. Revista Clase Empresarial, n. 54, Bogotá: Legis Editores. 
Gonczi, A. y Athanasou, J. (1996). Instrumentación de la educación basada en competencias. Perspectiva de la teoría y la práctica en Australia, México: Limusa.

Luna Scott, C. (2015). El futuro del aprendizaje ¿Qué tipo de aprendizaje se necesita en el siglo XXI? Revista Investigación y Prospectiva en Educación, n. 15, París: UNESCO.

Méndez, A.G. y Salas, Morales J. F. (2003). Desarrollo de habilidades y capacidades intelectuales. Serie Apoyo Didáctico, n. 5. México: UNAM-ENTS.

OCDE (1999). Proyectos sobre Competencias en el Contexto de la OCDE. Análisis de base teórica y conceptual, Suiza: OCDE.

OCDE (2010). Habilidades y competencias del siglo XXI para los aprendices del nuevo milenio en los países de la OCDE, París: OCDE.

Ouellet, A. (2000). La evaluación informativa al servicio de las competencias. Revista Escuela Administración de Negocios, n. 41, Colombia: EAN.

OECD (2016). Trends Shaping Education, París: OCDE.

Otero, J.M. y Luengo Orcajo, F. (Coords.) (2011). Teoría y práctica de las competencias básicas. Crítica y fundamentos, Barcelona: Graó.

Perrenoud, P. (2004). Diez nuevas competencias para enseñar. Barcelona: Graó.

Peters, Guy B. (2003). El nuevo institucionalismo: Teoría institucional en ciencia política. Barcelona: Gedisa.

RAE. (2001). Diccionario de la Lengua Española. España. En: http:/ / www.rae.es/recursos / diccionarios/drae

Rivas, P. (2004). La formación docente, realidad y retos en la sociedad del conocimiento. Revista Educere. Año 8, n. 24. Venezuela: Universidad de los Andes. 
Tawil, S. y Cougoureux, M. (2013). Una mirada actual a la educación encierra un tesoro, París: UNESCO.

Tobón Tobón, S. (2005). Formación basada en competencias. Pensamiento complejo, diseño curricular y didáctica, Bogotá: ECOE, Ediciones.

Toribio Briñas, L. (2010). Las competencias básicas: el nuevo paradigma curricular en Europa, Foro de Educación, vol. 8, n. 12, Valencia, España.

Trianes Torres, M. V., y Gallardo Cruz, J. A (Coords.) (2008). Psicología de la educación y del desarrollo en contexto escolares. Madrid: Pirámide.

Vasco, C. E. (2003). Objetivos específicos, indicadores de logros y competencias ¿y ahora estándares?, Revista Educación y Cultura, n. 62, Bogotá: Centro de Estudios e Investigaciones Docentes, Federación Colombiana de Educadores.

Venegas Jiménez, P. (2011), Gestión de la Educación basada en competencias: elementos para su interpretación en el contexto de la Administración de la Educación, Revista Gestión de la Educación. Vol. 1, n. 1, Universidad de Costa Rica, enero-junio, 2011, Costa Rica. 